\title{
EXTENSION OF AUTOMORPHISMS OF RATIONAL SMOOTH AFFINE CURVES
}

\author{
Jérémy Blanc, Jean-Philippe Furter, and Pierre-Marie Poloni
}

\begin{abstract}
We provide the existence, for every complex rational smooth affine curve $\Gamma$, of a linear action of $\operatorname{Aut}(\Gamma)$ on the affine 3 -dimensional space $\mathbb{A}^{3}$, together with a $\operatorname{Aut}(\Gamma)$ equivariant closed embedding of $\Gamma$ into $\mathbb{A}^{3}$. It is not possible to decrease the dimension of the target, the reason for this obstruction is also precisely described.
\end{abstract}

14R20, $14 \mathrm{H} 45$

\section{Introduction}

Throughout this article, all varieties are algebraic varieties defined over the field $\mathbb{C}$ of complex numbers. The affine (resp. projective) $n$-space is denoted by $\mathbb{A}^{n}$ (resp. $\mathbb{P}^{n}$ ).

It is well known that any smooth affine variety $X$ of dimension $n$ admits a closed embedding into $\mathbb{A}^{m}$, when $m \geq 2 n+1$ [12, Theorem 1]. If moreover $m \geq 2 n+2$, then, by a result of Nori, Srinivas and Kaliman (see [12 and [8]), any two closed embeddings $\iota, \iota^{\prime}: X \rightarrow \mathbb{A}^{m}$ are equivalent in the sense that there exists $f \in \operatorname{Aut}\left(\mathbb{A}^{m}\right)$ such that $\iota^{\prime}=f \circ \iota$.

In particular, if $\iota: X \rightarrow \mathbb{A}^{m}$ is a closed embedding of a smooth affine variety of dimension $n$ into some affine space of dimension $m \geq 2 n+2$, then it follows that every automorphism $\varphi$ of $X$ extends to an automorphism of the ambient space $\mathbb{A}^{m}$, since the two embeddings $\iota \circ \varphi$ and $\iota$ are equivalent.

However, Derksen, Kutzschebauch and Winkelmann showed in 4 that it is not always possible to extend the group structure of $\operatorname{Aut}(X)$, i.e. to find a closed embedding $\iota: X \rightarrow \mathbb{A}^{m}$ and an action of $\operatorname{Aut}(X)$ on $\mathbb{A}^{m}$ that restricts on $X$ to the action of $\operatorname{Aut}(X)$ on it. More precisely, they proved that there does not exist, for any integer $m$, any injective group homomorphism from $\operatorname{Aut}\left(\mathbb{C}^{*} \times \mathbb{C}^{*}\right) \cong \mathrm{GL}_{2}(\mathbb{Z}) \ltimes\left(\mathbb{C}^{*}\right)^{2}$ to the group Diff $\left(\mathbb{R}^{m}\right)$ of diffeomorphisms of $\mathbb{R}^{m}$.

Recall that, if $G$ is an algebraic group acting on an affine variety $X$, then $X$ admits a $G$-equivariant closed embedding into a finite dimensional $G$-module (see [2, Proposition 1.12 , p. 56]). In particular, there exist, for every smooth affine curve $\Gamma$, a linear action of $\operatorname{Aut}(\Gamma)$ on an affine space $\mathbb{A}^{m}$ and a $\operatorname{Aut}(\Gamma)$-equivariant closed embedding of $\Gamma$ into $\mathbb{A}^{m}$. A natural question is then to find the smallest possible $m$.

In this article, we settle the case of rational smooth affine curves. In this setting, the proof of Borel only gives the embedding dimension $m=2 \cdot|\operatorname{Aut}(\Gamma)|$, when the automorphism group $\operatorname{Aut}(\Gamma)$ is finite. However, our main result shows that it is already possible to obtain $m=3$ :

\footnotetext{
The authors gratefully acknowledge support by the Swiss National Science Foundation Grant "Birational Geometry" PP00P2_128422/1 and by the French National Research Agency Grant "BirPol", ANR-11-JS01-004-01.
} 
Theorem 1. Every rational smooth affine curve $\Gamma$ admits an Aut $(\Gamma)$-equivariant closed embedding into the affine space $\mathbb{A}^{3}$. Furthermore, there exist such embeddings for which the action of $\operatorname{Aut}(\Gamma)$ on $\mathbb{A}^{3}$ is linear.

It is easy to construct closed embeddings into the affine plane $\mathbb{A}^{2}$ for all rational smooth affine curves $\Gamma$. But it is of course not possible in general to ask for Aut $(\Gamma)$ equivariant embeddings into $\mathbb{A}^{2}$. Indeed, there exist rational smooth affine curves whose automorphism groups are isomorphic to the alternating group $\mathfrak{A}_{4}$, to $\mathfrak{A}_{5}$, or to the symmetric group $\mathfrak{S}_{4}$ (see Section 6) and it is well known that the group $\mathfrak{A}_{4}$ has no faithful representation of dimension two. Since all finite subgroups of Aut $\left(\mathbb{A}^{2}\right)$ are linearizable, it follows that we cannot embed equivariantly such a curve into the plane, even if we allow non linear actions on $\mathbb{A}^{2}$.

In fact, we establish stronger impossibility statements showing that it would be also too optimistic in general to look for closed embeddings into $\mathbb{A}^{2}$ in such a way that every single automorphism of the curve extends to an automorphism of the ambient space (see Corollary 2.6).

Theorem 2. There exist rational smooth affine curves $\Gamma$ with $\operatorname{Aut}(\Gamma) \neq 1$ and such that for every closed embedding of $\Gamma$ into $\mathbb{A}^{2}$, the identity on $\Gamma$ is its only automorphism that extends to an automorphism of $\mathbb{A}^{2}$.

Let us also emphasize that Theorem 1 cannot be generalized to all smooth affine curves. Actually, there even exist, for every natural number $n$, smooth affine curves $\Gamma$ which do not admit any Aut( $\Gamma)$-equivariant closed embedding into $\mathbb{A}^{n}$.

To see this, recall that every finite group $G$ is equal to the automorphism group of a smooth projective curve, and thus of an affine one [7, and take a smooth affine curve $\Gamma_{n}$ whose automorphism group is isomorphic to $(\mathbb{Z} / 2 \mathbb{Z})^{n+1}$. Then, $\Gamma_{n}$ does not admit any $\operatorname{Aut}\left(\Gamma_{n}\right)$-equivariant embedding into $\mathbb{A}^{n}$, because $(\mathbb{Z} / 2 \mathbb{Z})^{n+1}$ does not act faithfully on $\mathbb{A}^{n}$. Indeed, by Smith theory, the action of a finite $p$-group on $\mathbb{A}^{n}$ has always a fixed point (see e.g. [3, Th. 7.11, p 145], 9, p. 204], or [4, Proposition 1]) and the induced tangential (linear) representation at that fixed point should be faithful too (see e.g. 4, Lemma 4]).

It would however be interesting to know what happens in the case of smooth affine curves of genus 1 . Sathaye proved in 10 that such curves admit closed embeddings into $\mathbb{A}^{2}$. Nevertheless, we do not know what is the minimal $m$ (if it exists) such that every smooth affine curve $\Gamma$ of genus 1 admits an $\operatorname{Aut}(\Gamma)$-equivariant closed embedding into $\mathbb{A}^{m}$.

The article is organized as follows.

Section 2 concerns embeddings of rational smooth affine curves into the affine plane. We give examples of automorphisms of such curves that do not extend, and prove Theorem 2 (see Corollary 2.6).

Section 3 is devoted to the study of embeddings of smooth rational curves into $\mathbb{A}^{3}$ whose images are contained in a hyperplane. We prove that they are all equivalent and thus that any two closed embeddings of a rational smooth affine curve into $\mathbb{A}^{2}$ become equivalent, when seen as embeddings in $\mathbb{A}^{3}$ (Proposition 3.1). This answers a question of Bhatwadekar and Srinivas in this case.

In section 4 we realize every non-empty subset of $\mathbb{P}^{1}$ that is invariant by a subgroup $H$ of $\operatorname{Aut}\left(\mathbb{P}^{1}\right)$ as the fixed-point set of a $H$-equivariant endomorphism of $\mathbb{P}^{1}$ 
(Corollary 4.4). This result is used in Section 5 to prove Theorem1(see Theorem 5.2). Explicit formulas are given in Section [6

\section{Embeddings of rational smooth affine curves into the plane}

Let us recall that every rational smooth affine curve $\Gamma$ is isomorphic to $\mathbb{P}^{1} \backslash \Lambda$, where $\Lambda$ is a finite set of $r \geq 1$ points.

In particular, it admits a closed embedding into $\mathbb{A}^{2}$. Indeed, $\Gamma$ can also be seen as the complement in $\mathbb{A}^{1}$ of a finite number (possibly zero) of points and we can consider the closed embedding $\tau: \Gamma \rightarrow \mathbb{A}^{2}$ given by $x \mapsto\left(x, \frac{1}{P(x)}\right)$, where $P \in \mathbb{C}[x]$ is a polynomial whose roots are exactly the removed points. Note that the image of $\tau$ is the curve of $\mathbb{A}^{2}$ defined by the equation $P(x) y=1$.

Moreover, the automorphism group $\operatorname{Aut}(\Gamma)$ of the curve $\Gamma=\mathbb{P}^{1} \backslash \Lambda$ is equal to the group of automorphisms of $\mathbb{P}^{1}$ that preserve the set $\Lambda$. This gives a group homomorphism from $\operatorname{Aut}(\Gamma)$ to the symmetric group $\operatorname{Sym}_{r}$. Note that this homomorphism is injective if and only if $r \geq 3$.

If $r$ is equal to 1 or 2 , then $\Gamma$ is isomorphic to $\mathbb{A}^{1}$ or $\mathbb{A}^{1} \backslash\{0\}$, and its automorphism group is $\mathbb{C}^{*} \ltimes \mathbb{C}$ or $\{ \pm 1\} \ltimes \mathbb{C}^{*}$ respectively. If $r \geq 3$, then $\operatorname{Aut}(\Gamma)$ is a finite group.

The Abhyankar-Moh-Suzuki theorem claims that all closed embeddings of $\mathbb{A}^{1}$ into $\mathbb{A}^{2}$ are equivalent to the one given by $t \mapsto(t, 0)$. This implies that every automorphism of an affine line embedded into $\mathbb{A}^{2}$ extends to an automorphism of the ambiant space. If $r \geq 2$ we can on the contrary construct embeddings of the curve $\Gamma$ which do not have this property. Actually, we can choose embeddings such that, except the identity, no automorphisms of $\Gamma$ extend.

Lemma 2.1. Let $\Gamma=\mathbb{A}^{1} \backslash \Delta$, where $\Delta$ is a non-empty finite set. Then, there exist infinitely many non-equivalent closed embeddings $\iota: \Gamma \rightarrow \mathbb{A}^{2}$ such that the identity is the only automorphism of $\mathbb{A}^{2}$ that preserves $\iota(\Gamma)$.

Proof. We can assume that $\Delta=\left\{0, a_{1}, \ldots, a_{m}\right\}$, where $a_{1}, \ldots, a_{m} \in \mathbb{C} \backslash\{0,1\}, m \geq 0$. For every $k \geq 2$, we denote by $\iota_{k}: \Gamma \rightarrow \mathbb{A}^{2}$ the embedding given by

$$
x \mapsto\left(x, \frac{x-1}{x^{k} \prod_{i=1}^{m}\left(x-a_{i}\right)}\right) .
$$

It induces an isomorphism between $\Gamma$ and the curve $\iota_{k}(\Gamma)$ defined by the equation

$$
x=y x^{k} \prod_{i=1}^{m}\left(x-a_{i}\right)+1 .
$$

We first remark that any automorphism of $\mathbb{A}^{2}$ that sends $\iota_{k}(\Gamma)$ onto a curve of degree at most $\operatorname{deg}(\iota(\Gamma))=k+m+1$ is necessarily affine. Indeed, if $f:(x, y) \mapsto$ $\left(f_{1}(x, y), f_{2}(x, y)\right)$ is the inverse of such an automorphism, we get:

$$
\operatorname{deg}\left(f_{1}-f_{2}\left(f_{1}\right)^{k} \prod_{i=1}^{m}\left(f_{1}-a_{i}\right)-1\right)=(k+m) \operatorname{deg} f_{1}+\operatorname{deg} f_{2} \leq k+m+1 .
$$

This implies that $\operatorname{deg}\left(f_{1}\right)=\operatorname{deg}\left(f_{2}\right)=1$, i.e. that $f$ (and its inverse too) is affine. In particular, all above embeddings are non-equivalent. We now show that the identity is the only affine automorphism of $\mathbb{A}^{2}$ that preserves the curve $\iota_{k}(\Gamma)$. 
Any such automorphism extends to an automorphism $\tau$ of $\mathbb{P}^{2}$ preserving the line at infinity given by $z=0$ and the curve of equation

$$
x z^{k+m}-y x^{k} \prod_{i=1}^{m}\left(x-a_{i} z\right)-z^{k+m+1}=0 .
$$

On the line at infinity we get the two points $[0: 1: 0]$ and $[1: 0: 0]$. The point $[1: 0: 0]$ is smooth with tangent $y=0$ and the point $[0: 1: 0]$ is singular with tangent cone given by $x^{k} \prod_{i=1}^{m}\left(x-a_{i} z\right)=0$. Hence, both lines $x=0$ and $y=0$ are invariant. Therefore, $\tau$ is given by a diagonal automorphism of the form $[x: y: z] \mapsto[\mu x: \nu y: z]$, $\mu, \nu \in \mathbb{C}^{*}$. Replacing in the equation yields $\mu=\nu=1$.

The curves $\mathbb{A}^{1}$ and $\mathbb{A}^{1} \backslash\{0\}$ admit closed embeddings into $\mathbb{A}^{2}$ such that all their automorphisms extend to automorphisms of $\mathbb{A}^{2}$. Consider for example the curves of equations $y=0$ and $x y=1$. However, it is no longer true for the curve $\mathbb{A}^{1} \backslash\{0,1\}$.

Proposition 2.2. Let $\Gamma=\mathbb{A}^{1} \backslash\{0,1\}$. For every closed embedding $\tau: \Gamma \rightarrow \mathbb{A}^{2}$, there exists an automorphism of $\Gamma$ that does not extend to $\mathbb{A}^{2}$.

Before proving this statement, let us recall the following classical result (see e.g. 6. Theorem 2]).

Lemma 2.3. Every finite subgroup of $\operatorname{Aut}\left(\mathbb{A}^{2}\right)$ is conjugate to a subgroup of $\mathrm{GL}(2, \mathbb{C})$.

Proof of Proposition 2.2. Note that the group of automorphisms of $\Gamma$ is the group $\mathrm{Sym}_{3}$ of permutations of a set of three elements, corresponding to the three points "at infinity", i.e. the points of $\mathbb{P}^{1} \backslash \iota(\Gamma)$, where $\iota$ is any (open) embedding of $\Gamma$ in $\mathbb{P}^{1}$. It is generated by the automorphisms $\rho: x \mapsto 1 /(1-x)$ and $\sigma: x \mapsto 1-x$ and we have

$$
\operatorname{Aut}(\Gamma)=\left\langle\sigma, \rho \mid \sigma^{2}=\rho^{3}=1, \sigma \rho \sigma^{-1}=\rho^{-1}\right\rangle=\operatorname{Sym}_{3} .
$$

Suppose for contradiction that there exists a closed embedding $\tau: \Gamma \rightarrow \mathbb{A}^{2}$ for which every automorphism of $\Gamma$ extends. Since the identity is the only automorphism of $\mathbb{A}^{2}$ that restricts to the identity on a closed curve isomorphic to $\mathbb{A}^{1} \backslash\{0,1\}$ (see Lemma 2.4 below), we would have a subgroup $G \subset \operatorname{Aut}\left(\mathbb{A}^{2}\right)$ isomorphic to $\mathrm{Sym}_{3}$ whose restriction to $\tau(\Gamma)$ yields Aut $(\Gamma)$.

We now prove that this is impossible. First, recall that $G$ is conjugate to a subgroup of $\operatorname{GL}(2, \mathbb{C})$ (see Lemma 2.3 above). Then, one easily checks that $G$ is conjugate to the subgroup $G^{\prime}$ of $\mathrm{GL}(2, \mathbb{C})$ generated by

$$
\hat{\rho}:(x, y) \mapsto(y,-x-y) \text { and } \hat{\sigma}:(x, y) \mapsto(y, x) .
$$

We let $f \in \operatorname{Aut}\left(\mathbb{A}^{2}\right)$ be an automorphism such that $f G f^{-1}=G^{\prime}$ and we consider the embedding $\hat{\tau}=f \circ \tau$ of $\Gamma$ in $\mathbb{A}^{2}$. The automorphism group of $\Gamma$ extends then to $G^{\prime}$ for this embedding.

Remark that the set $\left\{\omega \mid \omega^{2}-\omega+1=0\right\} \subset \Gamma$, which is the set of fixed points of $\rho$, is an orbit of size 2 of $\operatorname{Aut}(\Gamma)$. But one checks that $G^{\prime} \subset \mathrm{GL}(2, \mathbb{C})$ does not have any orbit of size 2 in the affine plane $\mathbb{A}^{2}$. This gives a contradiction.

Lemma 2.4. The set of fixed points of a plane polynomial automorphism is either a finite set of points (possibly empty), a finite disjoint union of subvarieties isomorphic to $\mathbb{A}^{1}$, or the whole plane. 
Proof. Using the amalgamated structure of $\operatorname{Aut}\left(\mathbb{A}^{2}\right)$, it is observed in 5 that a plane polynomial automorphism is conjugate either to a triangular automorphism $(x, y) \mapsto$ $(a x+p(y), b y+c)$ with $a, b, c \in \mathbb{C}$ and $p(y) \in \mathbb{C}[y]$, or to some cyclically reduced element (see [11, I.1.3] or [5, p. 70] for the definition of a cyclically reduced element). In the first case, an obvious computation shows that the set of fixed points is either empty, a point, a finite disjoint union of subvarieties isomorphic to $\mathbb{A}^{1}$, or the whole plane. In the second case, by [5, Theorem 3.1], the set of fixed points is a non-empty finite set of points.

Using tools of birational geometry, we can actually specify the statement of Proposition 2.2. Indeed, Theorem 2.5 below shows that there is no closed embedding of the curve $\mathbb{A}^{1} \backslash\{0,1\}$ into $\mathbb{A}^{2}$ such that the automorphism $\rho: x \mapsto 1 /(1-x)$ extends to an automorphism of the affine plane.

Before we state this result, let us recall that any automorphism $f$ of $\mathbb{P}^{1}$ of finite order $n>1$ is conjugate to $[x: y] \mapsto[x: \xi y]$, where $\xi$ is a primitive $n$-th root of unity. In particular, it has the following properties:

(1) the automorphism $f$ fixes exactly two points of $\mathbb{P}^{1}$;

(2) all other orbits under the action of $f$ have size $n$.

Thus, the following holds for every automorphism $g \in \operatorname{Aut}(\Gamma)$ of order $n>1$ of a rational smooth curve $\Gamma$.

(1) The automorphism $g$ fixes 0,1 or 2 points of $\Gamma$;

(2) all other orbits under the action of $g$ have size $n$.

Theorem 2.5. Let $\Gamma$ be a rational smooth affine curve and let $g \in \operatorname{Aut}(\Gamma)$ be an automorphism.

(1) If $g$ fixes at most one point of $\Gamma$, there is a closed embedding $\tau: \Gamma \rightarrow \mathbb{A}^{2}$ such that $g$ extends to an automorphism of $\mathbb{A}^{2}$.

(2) If $g$ is of finite order $n>1$ with $n$ odd and if it fixes exactly two points of $\Gamma$, then there is no closed embedding $\tau: \Gamma \rightarrow \mathbb{A}^{2}$ such that $g$ extends to an automorphism of $\mathbb{A}^{2}$.

Proof. (1) Let $P \in \mathbb{C}[x]$ be a non-zero polynomial such that $\Gamma$ is isomorphic to $\mathbb{A}^{1} \backslash\left\{x \in \mathbb{A}^{1} \mid P(x)=0\right\}$. Let $g \in \operatorname{Aut}(\Gamma)$ be an automorphism that fixes at most one point of $\Gamma$. Let us denote also by $g$ its extension as an automorphism of $\mathbb{P}^{1}$. We can assume that $g$ fixes the point of $\mathbb{P}^{1}$ at infinity, so that it is of the form $x \mapsto a x+b$, for some $a \in \mathbb{C}^{*}$ and $b \in \mathbb{C}$. Moreover $P(a x+b)=\mu P(x)$ for some $\mu \in \mathbb{C}^{*}$.

When we embed $\Gamma$ into $\mathbb{A}^{2}$ via the map $x \mapsto\left(x, \frac{1}{P(x)}\right)$, the automorphism $g$ extends to $(x, y) \mapsto\left(a x+b, \mu^{-1} y\right)$.

(2) Let $g \in \operatorname{Aut}(\Gamma)$ be of finite order $n>1$ with $n$ odd such that it fixes 2 points of $\Gamma$. Suppose, for contradiction, that there exists a closed embedding $\tau: \Gamma \rightarrow \mathbb{A}^{2}$ for which $g$ extends to an automorphism $h$ of $\mathbb{A}^{2}$. Since $g$ has finite order $n$, the automorphism $h^{n} \in \operatorname{Aut}\left(\mathbb{A}^{2}\right)$ fixes pointwise the curve $\tau(\Gamma)$. Because $g$ fixes two points of $\Gamma, \tau(\Gamma)$ is not isomorphic to $\mathbb{A}^{1}$, hence $h^{n}$ is trivial by Lemma 2.4.

Recall that every automorphism of $\mathbb{A}^{2}$ of finite order is conjugate to a linear one (Lemma 2.3). Thus, there exists $f \in \operatorname{Aut}\left(\mathbb{A}^{2}\right)$ such that $\hat{h}=f \circ h \circ f^{-1}$ is linear. Moreover, the automorphism $g \in \operatorname{Aut}(\Gamma)$ extends to $\hat{h}$, when we consider the embedding $\hat{\tau}=f \circ \tau: \Gamma \rightarrow \mathbb{A}^{2}$. 
The linear automorphism $\hat{h}$ extends to an automorphism of $\mathbb{P}^{2}$, and the closure of $\hat{\tau}(\Gamma)$ in $\mathbb{P}^{2}$ is a projective rational curve $C$, having all its singular points on the line at infinity $L=\mathbb{P}^{2} \backslash \mathbb{A}^{2}$.

If $C$ is smooth, it is isomorphic to $\mathbb{P}^{1}$. Hence, it is a conic or a line, and thus intersects $L$ into 1 or 2 points, which contradicts the fact that $g$ acts on $C$ with order $n>2$ and with no fixed point at infinity. This implies that $C$ is singular.

Denote by $\eta_{1}: X_{1} \rightarrow \mathbb{P}^{2}$ the blow-up of the points of $\mathbb{P}^{2}$ that are singular points of $C$, and write $C_{1} \subset X_{1}$ the strict transform of $C$ in $X_{1}$. If $C_{1}$ is singular, we denote by $\eta_{2}: X_{2} \rightarrow X_{1}$ the blow-up of the points of $X_{1}$ that are singular points of $C_{1}$, and write $C_{2}$ the strict transform of $C_{1}$ in $X_{2}$. We continue like this until we end with a smooth curve $C_{m} \subset X_{m}$ such that the intersection of $C_{m}$ with all curves contracted by $\eta_{1} \eta_{2} \ldots \eta_{m}$ is transversal. Note that $C_{m}$ is isomorphic to $\mathbb{P}^{1}$. For $i=1, \ldots, m$, the lift of $\hat{h}$ yields an automorphism $h_{i}$ of $X_{i}$ which preserves the curve $C_{i}$. It also preserves the pull-back of $\mathbb{A}^{2}$ in $X_{i}$, which is again isomorphic to $\mathbb{A}^{2}$.

For $i=1, \ldots, m$, we denote by $\mathcal{B}_{i} \subset C_{i}$ the (finite) set of points not lying in $\mathbb{A}^{2}$. Each point $p \in \mathcal{B}_{i}$ has a multiplicity $m(p)$ as a point of $C_{i}$. This multiplicity is a positive integer and it is equal to 1 if and only if $C_{i}$ is smooth at this point $p$. Denote by $\mathcal{B}_{0}$ the set of points of $C_{0}=C \subset X_{0}=\mathbb{P}^{2}$ not lying in $\mathbb{A}^{2}$ and let us use the same notation as above for the multiplicities of the points of $\mathcal{B}_{0}$.

Writing $d$ the degree of $C \subset \mathbb{P}^{2}$, the geometric genus of $C$ can be computed with the following classical formula. (Note that it is equal to 0 , since $C$ is rational.)

$$
0=\frac{(d-1)(d-2)}{2}-\sum_{i=0}^{m} \sum_{p \in \mathcal{B}_{i}} \frac{m(p) \cdot(m(p)-1)}{2} .
$$

Let us now prove the following assertion by descending induction on $j \leq m$.

$(\diamond)$

Let $j \in\{1, \ldots, m\}$ and let $J \subset \mathcal{B}_{j}$ be an orbit under the action of $h_{j}$.

Then $m(p)=m\left(p^{\prime}\right)$ for all $p, p^{\prime} \in J$, and the integer $\sum_{p \in J} m(p)$ is a multiple of $\mathrm{n}$.

For $j=m$, the assertion $(\diamond)$ holds for all orbits $J \subset \mathcal{B}_{m}$. Indeed, $C_{m}$ is isomorphic to $\mathbb{P}^{1}$ and the action of $h_{m}$ on $\mathcal{B}_{m} \subset C_{m}$ is fixed-point-free, so all orbits have size $n$ and all multiplicities are equal to 1 .

Then, we can prove $(\diamond)$ for $j<m$, assuming it holds for every integer $k$ with $j+1 \leq k \leq m$. For this, let $J \subset \mathcal{B}_{j}$ be an orbit under the action of $h_{j}$ and let us denote by $m_{J}$ the multiplicity $m(p)$ of a point $p \in J$. Note that this multiplicity does not depend of the choice of $p$, since $h_{j}$ acts transitively on $J$.

If $m_{J}=1$, all points of $J$ are smooth, and so the pull-back by $\eta_{j+1}$ of $J$ consists of $|J|$ points of multiplicity $m_{j}=1$. This implies $\sum_{p \in J} m(p) \in n \mathbb{N}$, by induction hypothesis.

If $m_{J}>1$, then all points of $J$ are singular points of the curve $C_{j}$ and are thus blown-up by $\eta_{j+1}: X_{j+1} \rightarrow X_{j}$. The number $m_{J}$ is the multiplicity of the curve $C_{j}$ at the point $p \in J$. Denoting by $E_{p} \subset X_{j+1}$ the curve contracted by $\eta_{j+1}$ onto $p$, the number $m_{J}$ is the intersection number $E_{p} \cdot C_{j+1}$. This latter is equal to the sum of $m_{q}\left(E_{p}\right) \cdot m_{q}\left(C_{j+1}\right)$, where $q$ runs through all points infinitely near to $p$, and where 
$m_{q}\left(E_{p}\right)$ and $m_{q}\left(C_{j+1}\right)$ are the multiplicities of the strict transforms of $E_{p}$ and $C_{j+1}$ at $q$, respectively. Note that $m_{q}\left(E_{p}\right)$ is equal to 0 or 1 .

Therefore, the sum $\sum_{p \in J} m_{J}$ is equal to a sum of multiplicities of orbits in $\mathcal{B}_{k}$ for $k \geq j+1$. By induction hypothesis, it is a multiple of $n$. This achieves to prove $(\diamond)$.

In order to finish the proof, we will show how Equation (因) and Assertion (四) imply that the integers $\frac{(d-1)(d-2)}{2}$ and $d$ are both multiple of $n$. Since the greatest common divisor of $d$ and $\frac{(d-1)(d-2)}{2}$ is 1 or 2 , this will contradict the assumption $n>2$.

To show that $n$ divides $\frac{(d-1)(d-2)}{2}$, we decompose the sum of (因) according to orbits

$$
\frac{(d-1)(d-2)}{2}=\sum_{j=0}^{m} \sum_{J \subset \mathcal{B}_{j}} \sum_{p \in J} \frac{m(p) \cdot(m(p)-1)}{2} .
$$

By Assertion (四), the multiplicities $m(p)$ are all equal among the same orbit $J$, so $\sum_{p \in J} m(p) \cdot(m(p)-1)$ is a multiple of $\sum_{p \in J} m(p)$, which is a multiple of $n$ by (因). Since $n$ is odd, $\sum_{p \in J} \frac{m(p) \cdot(m(p)-1)}{2}$ is also a multiple of $n$, and so is $\frac{(d-1)(d-2)}{2}$.

It remains to show that $d$ is also a multiple of $n$. For this, we observe that the intersection number $d=L \cdot C$ is the sum of multiplicities of all points of $C$ that belong to $L$, as proper or infinitely near points. Since $L$ is invariant under the extension of the affine automorphism $\hat{h}$, the union of these points decomposes into orbits of $h_{j}$ and the sum is then a multiple of $n$ by Assertion (四).

Corollary 2.6. There exist rational smooth affine curves $\Gamma$ with $\operatorname{Aut}(\Gamma) \neq 1$ and such that for every closed embedding of $\Gamma$ in $\mathbb{A}^{2}$, the identity on $\Gamma$ is its only automorphism which extends to an automorphism of $\mathbb{A}^{2}$.

Proof. Let $\omega=e^{2 i \pi / 3}$ and $a_{1}=1$. Let $a_{2}, \ldots, a_{k}$ be complex numbers algebraically independent over $\mathbb{Q}$. We consider the curve $\Gamma=\mathbb{P}^{1} \backslash \Lambda$, where $\Lambda$ is the following set of $3 k$ points

$$
\Lambda=\left\{\left[a_{i} \omega^{j}: 1\right] \mid i=1, \ldots, k, j=0, \ldots, 2\right\} .
$$

The map $h:[x: y] \mapsto[x: \omega y]$ is obviously an automorphism of $\Gamma$. We will now prove that it generates the whole automorphism group $\operatorname{Aut}(\Gamma)$ if $k \geq 3$. This will conclude the proof, since $h$ and $h^{2}$ do not extend to automorphisms of $\mathbb{A}^{2}$ by Theorem 2.5.

Let $g \in \operatorname{Aut}(\Gamma)$ be an automorphism of $\Gamma$. It extends to an automorphism of $\mathbb{P}^{1}$ that preserves the set $\Lambda$. Let us denote this latter also by $g$.

Consider the 4-tuple $V=\left([1: 1],[\omega: 1],\left[\omega^{2}: 1\right],\left[a_{2}: 1\right]\right)$. Since $a_{2}, \ldots, a_{k}$ are algebraically independent over $\mathbb{Q}$, the image of $V$ by $g$ is a 4 -tuple of points contained in the set

$$
S=\left\{[1: 1],[\omega: 1],\left[\omega^{2}: 1\right],\left[a_{2}: 1\right],\left[a_{2} \omega: 1\right],\left[a_{2} \omega^{2}: 1\right]\right\} .
$$

Indeed, the cross-ratio of $g(V)$ must be equal to the cross-ratio of $V$, i.e. to $\omega(\omega-$ $\left.a_{2}\right) /\left(a_{2}-1\right)$.

The same argument with the 4 -tuple $\left([1: 1],[\omega: 1],\left[\omega^{2}: 1\right],\left[a_{3}: 1\right]\right)$ allows us to conclude that $g$ preserves the set $\left\{[1: 1],[\omega: 1],\left[\omega^{2}: 1\right]\right\}$. Therefore, $g$ is either a power of $h$, or it is one of the maps $\varphi_{i}:[x: y] \mapsto\left[y: x \omega^{i}\right]$ with $i=0 \ldots 2$.

Finally, note that $g$ cannot be one of the $\varphi_{i}$ 's, since $\varphi_{i}$ sends the point $\left[a_{2}: 1\right]$ onto the point $\left[\frac{1}{a_{2} \omega^{i}}: 1\right]$, which does not belong to the set $S$. 
Remark 2.7. The proof of Corollary 2.6 shows that if $k \geq 3$ and if the set $\Lambda \subset \mathbb{P}^{1}$ is general among all sets of distinct $3 k$ points invariant by the map $[x: y] \mapsto[x: \omega y]$, then for all closed embeddings of the curve $\Gamma=\mathbb{P}^{1} \backslash \Lambda$ into $\mathbb{A}^{2}$, the identity is the only automorphism of $\Gamma$ that extends to an automorphism of $\mathbb{A}^{2}$.

On the contrary, when $k \leq 2$, every such curve $\Gamma$ admits an automorphism of order 2 and Proposition 2.8 below implies then that this latter extends to an automorphism of $\mathbb{A}^{2}$ for a well-chosen closed embedding of $\Gamma$ into $\mathbb{A}^{2}$.

Proposition 2.8. Let $\Gamma$ be a rational smooth affine curve and let $\sigma \in \operatorname{Aut}(\Gamma)$ be an automorphism of $\Gamma$ of order 2. There exists a closed embedding of $\Gamma$ in $\mathbb{A}^{2}$ and an automorphism $\hat{\sigma} \in \operatorname{Aut}\left(\mathbb{A}^{2}\right)$ of order 2 whose restriction to $\Gamma$ yields $\sigma$.

Proof. Let $\Gamma=\mathbb{P}^{1} \backslash \Lambda$, where $\Lambda$ is a finite set of points. Let us denote by $\sigma$ the extension of the automorphism $\sigma \in \operatorname{Aut}(\Gamma)$ as an automorphism of $\mathbb{P}^{1}$. If it fixes at most one point of $\Lambda$, the result follows from Theorem 2.5

We can thus assume that the two points of $\mathbb{P}^{1}$ fixed by (the extension of) $\sigma$ belong to $\Gamma$. Let $p$ be a point of $\Lambda$. Its orbit $\{p, \sigma(p)\}$ is then contained in $\Lambda$. Let $C$ be the curve $C=\mathbb{P}^{1} \backslash\{p, \sigma(p)\}$. Note that $C$ is isomorphic to $\mathbb{A}^{1} \backslash\{0\}$ and that $\sigma$ restricts to an automorphism of $C$. Remark that all automorphisms of $\mathbb{A}^{1} \backslash\{0\}$ of order 2 with two fixed points are conjugate to the automorphism $x \mapsto x^{-1} \in \operatorname{Aut}\left(\operatorname{Spec}\left(\mathbb{C}\left[x, x^{-1}\right]\right)\right)$. Therefore, there is a closed embedding $\tau: C \rightarrow \mathbb{A}^{2}$ whose image is the curve defined by the equation

$$
y^{2}-1=x^{2}
$$

and such that $\sigma \in \operatorname{Aut}(C)$ extends to the automorphism $\hat{\sigma}:(x, y) \mapsto(-x, y)$. Moreover, the curve $\tau(\Gamma)$ is then equal to a set of points of $\tau(C)$ satisfying that $\prod_{i=1}^{n}(y-$ $\left.a_{i}\right) \neq 0$, for some $n \geq 0$ and distinct $a_{1}, \ldots, a_{n} \in \mathbb{C} \backslash\{ \pm 1\}$.

Let $Y \subset \mathbb{A}^{2}$ be the closed curve defined by the equation

$$
y^{2}-1=x^{2} \cdot\left(\prod_{i=1}^{n}\left(y-a_{i}\right)\right)^{2} .
$$

Consider finally the birational transformation of $\mathbb{A}^{2}$ defined by

$$
(x, y) \rightarrow\left(\frac{x}{\prod_{i=1}^{n}\left(y-a_{i}\right)}, y\right),
$$

which restricts to an isomorphism between $\tau(\Gamma)$ and $Y$. Since it commutes with the automorphism $(x, y) \mapsto(-x, y)$, this yields the result.

\section{Planar embeddings in the space}

The following question of Bhatwadekar and Srinivas is asked at the end of [12]: are any two embeddings of a smooth affine curve in $\mathbb{A}^{2}$ equivalent, when considered as embeddings in $\mathbb{A}^{3}$ ?

The next result answers positively for the case of rational smooth affine curves.

Proposition 3.1. Let $\Gamma$ be a rational smooth affine curve. 
(1) If $\tau_{1}, \tau_{2}: \Gamma \rightarrow \mathbb{A}^{3}$ are two closed embeddings whose images are contained in a hyperplane (planar embeddings in the space), there exists an automorphism $\alpha \in \operatorname{Aut}\left(\mathbb{A}^{3}\right)$ such that $\tau_{2}=\alpha \circ \tau_{1}$, i.e. any two planar embeddings in the space are equivalent.

(2) In particular, fixing a planar embedding $\Gamma \rightarrow \mathbb{A}^{3}$, every automorphism of $\Gamma$ extends to $\mathbb{A}^{3}$.

Proof. Let $\Gamma=\mathbb{A}^{1} \backslash\left\{x \in \mathbb{A}^{1} \mid P(x)=0\right\}$, where $P \in \mathbb{C}[x]$ is a polynomial with simple roots. Note that the coordinate ring of $\Gamma$ is $\mathbb{C}[\Gamma]=\mathbb{C}\left[x, \frac{1}{P(x)}\right]$ and recall that the map $x \mapsto\left(x, \frac{1}{P(x)}\right)$ defines a closed embedding of $\Gamma$ in $\mathbb{A}^{2}$. To prove the proposition, it suffices to prove that any planar embedding is equivalent to the one given by $x \mapsto\left(x, \frac{1}{P(x)}, 0\right)$.

Let $\tau: \Gamma \rightarrow \mathbb{A}^{3}$ be a planar embedding of $\Gamma$. We can compose $\tau$ with an affine automorphism $f_{1}$ of $\mathbb{A}^{3}$ and get an embedding $\tau_{2}=f_{1} \circ \tau: \Gamma \rightarrow \mathbb{A}^{3}$ of the form $x \mapsto(0, Q(x), R(x))$, where $Q, R \in \mathbb{C}(x)$ are rational functions without poles on $\Gamma$. Since $\tau_{2}$ is a closed embedding of the curve $\Gamma$, the equality $\mathbb{C}\left[x, \frac{1}{P(x)}\right]=\mathbb{C}[Q(x), R(x)]$ holds. In particular, there exists a polynomial $A \in \mathbb{C}[X, Y]$ such that $A(Q(x), R(x))=$ $x$. Now, we compose $\tau_{2}$ with the automorphism of $\mathbb{A}^{3}$ defined by $f_{2}(X, Y, Z)=$ $(X+A(Y, Z), Y, Z)$ and obtain the embedding $\tau_{3}: \Gamma \rightarrow \mathbb{A}^{3}$ given by

$$
\tau_{3}: x \mapsto(x, Q(x), R(x)) .
$$

Because of the equality $\mathbb{C}\left[x, \frac{1}{P(x)}\right]=\mathbb{C}[Q(x), R(x)]$, all zeros of $P(x)$ are poles of $a Q(x)+b R(x)$ for general complex numbers $a, b \in \mathbb{C}$. We can thus compose $\tau_{3}$ with a linear automorphism of the form $(X, Y, Z) \mapsto(X, a Y+b Z, Z)$ and get an embedding $\tau_{4}: \Gamma \rightarrow \mathbb{A}^{3}$ of the form

$$
\tau_{4}: x \mapsto\left(x, \frac{Q_{1}(x)}{Q_{2}(x)}, \frac{R_{1}(x)}{R_{2}(x)}\right),
$$

where $Q_{1}, Q_{2}, R_{1}, R_{2} \in \mathbb{C}[x]$ are polynomials such that $Q_{1}$ and $Q_{2}$ (resp. $R_{1}$ and $R_{2}$ ) have no common factor, and such that $P(x)$ divides $Q_{2}(x)$.

In particular, there exist two polynomials $U, V \in \mathbb{C}[x]$ such that $U Q_{1}+V P=1$. It follows

$$
\frac{1}{P}=\frac{U Q_{1}+V P}{P}=U \frac{Q_{1}}{P}+V=S U \frac{Q_{1}}{Q_{2}}+V,
$$

where $S \in \mathbb{C}[x]$ satisfies $P S=Q_{2}$.

This implies $\mathbb{C}\left[x, \frac{1}{P}\right] \subset \mathbb{C}\left[x, \frac{Q_{1}}{Q_{2}}\right]$ and thus

$$
\mathbb{C}\left[x, \frac{Q_{1}}{Q_{2}}, \frac{R_{1}}{R_{2}}\right]=\mathbb{C}\left[x, \frac{1}{P}\right]=\mathbb{C}\left[x, \frac{Q_{1}}{Q_{2}}\right] .
$$

Therefore, there exist polynomials $B, C \in \mathbb{C}[X, Y]$ such that $B\left(x, \frac{Q_{1}(x)}{Q_{2}(x)}\right)=\frac{1}{P(x)}-$ $\frac{R_{1}(x)}{R_{2}(x)}$ and $C\left(x, \frac{1}{P(x)}\right)=\frac{Q_{1}(x)}{Q_{2}(x)}$. Finally, we consider the automorphisms of $\mathbb{A}^{3}$ defined by $f_{4}(X, Y, Z)=(X, Y, Z+B(X, Y))$ and $f_{5}(X, Y, Z)=(X, Z, Y-C(X, Z))$. One checks that $f_{5} \circ f_{4} \circ \tau_{4}: \Gamma \rightarrow \mathbb{A}^{3}$ is the desired embedding $x \mapsto\left(x, \frac{1}{P(x)}, 0\right)$.

Note that the proof above is constructive. In particular, a planar embedding of a smooth rational curve $\Gamma$ in $\mathbb{A}^{3}$ and an automorphism $\varphi$ of $\Gamma$ being given, it allows us to construct an explicit automorphism of $\mathbb{A}^{3}$ which extends $\varphi$. 
Example 3.2. Let $\Gamma$ be the curve $\Gamma=\mathbb{A}^{1} \backslash\{0,1\}$ and let $\rho \in \operatorname{Aut}(\Gamma)$ be the automorphism of $\Gamma$ defined by $\rho(x)=1 /(1-x)$. We saw in Section 2 that there is no closed embedding of $\Gamma$ into $\mathbb{A}^{2}$ such that $\rho$ extends to an automorphism of $\mathbb{A}^{2}$. However, it extends to an automorphism of $\mathbb{A}^{3}$, when we consider the embedding $\tau: \Gamma \rightarrow \mathbb{A}^{3}$ defined by $x \mapsto(x, 1 / x(x-1), 0)$.

Following the proof of Proposition 3.1 we let $f_{1}, f_{2}, \ldots, f_{5}$ be the automorphisms of $\mathbb{A}^{3}$ defined by $f_{1}(X, Y, Z)=(Z, Y, X), f_{2}(X, Y, Z)=\left(X+Y+2-Y Z^{2}, Y, Z\right)$, $f_{3}(X, Y, Z)=(X, a Y+b Z, Z), f_{4}(X, Y, Z)=\left(X, Y, Z-\frac{1}{a b}[(b+(a-b) X)(Y-a X+\right.$ $\left.\left.2 a)-(a-b)^{2}\right](1+X)\right)$ and $f_{5}(X, Y, Z)=(X, Z, Y-a X+2 a+a Z+(b-a) X Z)$, where $a, b \in \mathbb{C}$ are general complex numbers.

Setting $F=f_{5} \circ f_{4} \circ \cdots \circ f_{1}$, one checks $F \circ \tau \circ \rho=\tau$. This implies that $F^{-1}$ is an extension of the automorphism $\rho \in \operatorname{Aut}(\Gamma)$.

Remark 3.3. To our knowledge, there is no known example of a smooth affine curve admitting two non-equivalent embeddings into $\mathbb{A}^{3}$. Paradoxically, we do not know any smooth affine curve such that all its embeddings into $\mathbb{A}^{3}$ are equivalent!

The case of the affine line is of particular interest. On one hand, all closed embeddings of $\mathbb{A}^{1}$ into $\mathbb{A}^{2}$ are equivalent by the famous Abhyankar-Moh-Suzuki theorem. On the other hand, all closed embeddings of $\mathbb{A}^{1}$ into $\mathbb{A}^{n}$ with $n \geq 4$ are also equivalent (see [12] or [8]).

\section{Actions of $\operatorname{SL}(2, \mathbb{C})$ on $\operatorname{End}\left(\mathbb{A}^{2}\right)$ and of $\operatorname{PGL}(2, \mathbb{C})$ on $\mathbb{P}^{1}$}

The aim of this section is to construct, for every non-empty subset $\Lambda$ of $\mathbb{P}^{1}$ that is invariant by a subgroup $H$ of $\operatorname{Aut}\left(\mathbb{P}^{1}\right)$, a $H$-equivariant endomorphism of $\mathbb{P}^{1}$ whose fixed-point set is equal to the set $\Lambda$ (Corollary 4.4). We will use this result later on to construct embeddings of every rational smooth affine curve into $\mathbb{A}^{3}$ in such a way that the whole automorphism group of the curve extends to a subgroup of $\operatorname{Aut}\left(\mathbb{A}^{3}\right)$.

For the rest of the paper we will consider the following actions of the group $\operatorname{SL}(2, \mathbb{C})$ on $\mathcal{O}\left(\mathbb{A}^{2}\right)=\mathbb{C}[x, y]$ and $\operatorname{End}\left(\mathbb{A}^{2}\right)=\mathbb{C}[x, y] \times \mathbb{C}[x, y]$.

$$
\begin{array}{ccc}
\mathrm{SL}(2, \mathbb{C}) \times \mathcal{O}\left(\mathbb{A}^{2}\right) & \rightarrow & \mathcal{O}\left(\mathbb{A}^{2}\right) \\
(g, P) & \mapsto & g \cdot P:=P \circ g^{-1}
\end{array}
$$

and

$$
\begin{array}{ccc}
\operatorname{SL}(2, \mathbb{C}) \times \operatorname{End}\left(\mathbb{A}^{2}\right) & \rightarrow & \operatorname{End}\left(\mathbb{A}^{2}\right) \\
(g, F) & \mapsto g \cdot F:=g \circ F \circ g^{-1} .
\end{array}
$$

Note that these actions come from the natural action of $\operatorname{SL}(2, \mathbb{C})$ on $\mathbb{A}^{2}$. Indeed, denote by $V$ the space $\mathbb{A}^{2}$ as a complex vector space of dimension 2 and identify the set of the linear forms on it as the dual space $V^{*}$. The action of $\operatorname{SL}(V)$ on $V$ yields actions on $V^{*}$, on the symmetric algebra $S\left(V^{*}\right)$ and on $S\left(V^{*}\right) \otimes V$. The natural isomorphisms between $S\left(V^{*}\right)$ and $\mathbb{C}[x, y]=\mathcal{O}\left(\mathbb{A}^{2}\right)$, and between $S\left(V^{*}\right) \otimes V$ and $\mathbb{C}[x, y] \times \mathbb{C}[x, y]=\operatorname{End}\left(\mathbb{A}^{2}\right)$, lead then to the $\mathrm{SL}(2, \mathbb{C})$-actions that we defined above.

Lemma 4.1. The map $\rho: \operatorname{End}\left(\mathbb{A}^{2}\right) \rightarrow \mathcal{O}\left(\mathbb{A}^{2}\right)$ defined by

$$
\begin{array}{clc}
\mathbb{C}[x, y] \times \mathbb{C}[x, y] & \rightarrow \mathbb{C}[x, y] \\
\left(f_{1}, f_{2}\right) & \mapsto f_{1} y-f_{2} x
\end{array}
$$

is $\mathrm{SL}(2, \mathbb{C})$-equivariant, when we consider the actions defined above. 
Proof. The result could of course be checked by direct computations, but let us mention that it also follows from the fact that $\rho$ corresponds to the morphism $S\left(V^{*}\right) \otimes V \rightarrow$ $S\left(V^{*}\right)$ given by the composition $\tau_{2} \circ \tau_{1}$, where $\tau_{1}$ and $\tau_{2}$ are the two following homomorphisms of $\mathrm{SL}(V)$-modules.

$$
\begin{aligned}
\tau_{1}: \quad S\left(V^{*}\right) \otimes V & \rightarrow S\left(V^{*}\right) \otimes V \otimes V^{*} \otimes V \\
p \otimes v & \mapsto p \otimes v \otimes \mathrm{id},
\end{aligned}
$$

where id denotes the identity element seen as an element of $V^{*} \otimes V=\operatorname{Hom}(V, V)$, and

$$
\begin{aligned}
\tau_{2}: \quad S\left(V^{*}\right) \otimes V \otimes V^{*} \otimes V & \rightarrow S\left(V^{*}\right) \\
p \otimes v_{1} \otimes v_{2}^{*} \otimes v_{3} & \mapsto \operatorname{det}\left(v_{1}, v_{3}\right)\left(p v_{2}^{*}\right) .
\end{aligned}
$$

Lemma 4.2. Let $G \subset \mathrm{SL}(2, \mathbb{C})$ be a finite subgroup of $\mathrm{SL}(2, \mathbb{C})$ and let $P \in \mathbb{C}[x, y]$. The following conditions are equivalent:

(1) The polynomial $P$ satisfies $P(0,0)=0$ and is fixed by $G$.

(2) There exists an endomorphism $F=\left(f_{1}, f_{2}\right)$ of $\mathbb{A}^{2}$ that is fixed by $G$ and such that $\rho(F)=f_{1} y-f_{2} x=P$.

Proof. Let $E_{P} \subset \operatorname{End}\left(\mathbb{A}^{2}\right)$ be the set

$$
E_{P}=\rho^{-1}(P)=\left\{\left(f_{1}, f_{2}\right) \in \mathbb{C}[x, y] \times \mathbb{C}[x, y] \mid f_{1} y-f_{2} x=P\right\} .
$$

This defines an affine subset of the $\mathbb{C}$-vector space $\operatorname{End}\left(\mathbb{A}^{2}\right)$, since the endomorphism $\left(\lambda f_{1}+(1-\lambda) f_{3}, \lambda f_{2}+(1-\lambda) f_{4}\right)$ belongs to $E_{P}$, for any $\left(f_{1}, f_{2}\right),\left(f_{3}, f_{4}\right) \in E_{P}$ and any $\lambda \in \mathbb{C}$. Moreover, $E_{P}$ is non-empty if and only if $P(0,0)=0$.

If $F \in \operatorname{End}\left(\mathbb{A}^{2}\right)$ is fixed by $G$ and belongs to $E_{P}$, then

$$
g \cdot P=g \cdot \rho(F)=\rho(g \cdot F)=\rho(F)=P
$$

hold for any $g \in G$. This shows $(2) \Rightarrow(1)$.

If $P$ is fixed by $G$, then the set $E_{P}$ is invariant by $G$, since

$$
\rho(g \cdot F)=g \cdot \rho(F)=g \cdot P=P
$$

hold for any $F \in E_{P}$ and $g \in G$.

Therefore, if $F$ belongs to $E_{P}$, then $\frac{1}{|G|} \sum_{g \in G} g \cdot F$ is an element of $E_{P}$ that is fixed by $G$. This shows (1) $\Rightarrow(2)$ and concludes the proof.

Proposition 4.3. Let $H \subset \operatorname{PGL}(2, \mathbb{C})=\operatorname{Aut}\left(\mathbb{P}^{1}\right)$ be a finite subgroup and set $G=$ $\pi^{-1}(H)$, where $\pi: \operatorname{SL}(2, \mathbb{C}) \rightarrow \operatorname{PGL}(2, \mathbb{C})$ is the canonical surjective map. Let $\Lambda \subset \mathbb{P}^{1}$ be a non-empty $H$-invariant finite subset.

(1) There exist homogeneous polynomials $f_{1}, f_{2} \in \mathbb{C}[x, y]$ of the same degree such that $\left(f_{1}, f_{2}\right)$ is an endomorphism of $\mathbb{A}^{2}$ fixed by $G$ and such that

$$
\Lambda=\left\{[x: y] \in \mathbb{P}^{1} \mid f_{1}(x, y) y-f_{2}(x, y) x=0\right\} .
$$

(2) The morphism $\delta: \mathbb{P}^{1} \rightarrow \mathbb{P}^{1}$ defined by

$$
[x: y] \mapsto\left[f_{1}(x, y): f_{2}(x, y)\right]
$$

is $H$-equivariant, for all pairs $\left(f_{1}, f_{2}\right)$ given by the statement (1) above. 
(3) There exist polynomials $f_{1}, f_{2}$ satisfying the statement (1) and also the extra property

$$
\Lambda=\left\{q \in \mathbb{P}^{1} \mid \delta(q)=q\right\} .
$$

This latter holds moreover for all pairs $\left(f_{1}, f_{2}\right)$ given by the statement (1), in the case where the set $\Lambda$ consists of exactly one orbit of $H$.

Proof. (1) We let $p \in \mathbb{C}[x, y]$ be the (unique up a nonzero constant) square-free homogeneous polynomial whose roots correspond to the points of $\Lambda$. Because $\Lambda$ is invariant by $H$, there exists a character $\chi: G \rightarrow \mathbb{C}^{*}$ such that

$$
p \circ g=\chi(g) p,
$$

for all $g \in G$. Since $G$ is finite, there exists a positive integer $d$ such that the polynomial $P=p^{d}$ is fixed by $G$.

By Lemma 4.2, there exists an endomorphism $\left(f_{1}, f_{2}\right) \in \mathbb{C}[x, y] \times \mathbb{C}[x, y]$ of $\mathbb{A}^{2}$ that is fixed by $G$ and such that $f_{1} y-f_{2} x=P$. Since $P$ is homogeneous and since the action of $G$ on $\operatorname{End}\left(\mathbb{A}^{2}\right)$ is linear and preserves the filtration by degrees, we can assume that $f_{1}$ and $f_{2}$ are homogeneous of the same degree. This proves (1).

Statement (2) follows directly from the fact that the endomorphism $\left(f_{1}, f_{2}\right)$ is fixed by $G$.

(3) Since $\delta$ is $H$-equivariant, its fixed-point set is invariant by $H$. Let us denote it by $\Omega_{\delta}$ and write $f_{1}=\alpha \tilde{f}_{1}$ and $f_{2}=\alpha \tilde{f}_{2}$, where $\alpha, \tilde{f}_{1}, \tilde{f}_{2}$ are homogeneous polynomials such that $\tilde{f}_{1}$ and $\tilde{f}_{2}$ have no common root in $\mathbb{P}^{1}$. Then, $\delta([x: y])=\left[\tilde{f}_{1}(x, y): \tilde{f}_{2}(x, y)\right]$ holds for all $[x: y] \in \mathbb{P}^{1}$. The set $\Omega_{\delta}=\left\{q \in \mathbb{P}^{1} \mid \delta(q)=q\right\}$ is thus the zero set of $\tilde{f}_{1} y-\tilde{f}_{2} x$. In particular, it is non-empty. Moreover, the equalities $P=f_{1} y-f_{2} x=$ $\alpha\left(\tilde{f}_{1} y-\tilde{f}_{2} x\right)$ imply that $\Omega_{\delta}$ is contained in $\Lambda$.

If $\Lambda$ consists of exactly one orbit of $H$, then $\Omega_{\delta}=\Lambda$ follows from the fact that $\Omega_{\delta}$ is invariant by $H$.

Let us now consider the general case, where $\Lambda$ consists of $r>1$ orbits of $H$ and write $\Lambda=\bigcup_{i=1}^{r} \Lambda_{i}$, where $\Lambda_{1}, \ldots, \Lambda_{r}$ are disjoint orbits of $H$. For each $i$, there exist, by the previous argument, homogeneous polynomials $f_{i, 1}, f_{i, 2}$ of the same degree such that the zero set of $P_{i}=f_{i, 1} y-f_{i, 2} x$ is equal to $\Lambda_{i}$ and such that the pair $\left(f_{i, 1}, f_{i, 2}\right)$ defines an endomorphism of $\mathbb{A}^{2}$ which is fixed by $G$.

Set

$$
g_{1}=\frac{1}{r} \sum_{i=1}^{r}\left(f_{i, 1} \prod_{j \neq i} P_{j}\right) \quad \text { and } \quad g_{2}=\frac{1}{r} \sum_{i=1}^{r}\left(f_{i, 2} \prod_{j \neq i} P_{j}\right) .
$$

Note that $g_{1}$ and $g_{2}$ are homogeneous of the same degree and satisfy the equality $g_{1} y-g_{2} x=\prod_{i=1}^{r} P_{i}$. Moreover, the endomorphism $\left(g_{1}, g_{2}\right) \in \operatorname{End}\left(\mathbb{A}^{2}\right)$ is fixed by $G$. In other words, it satisfies the statement (1) of the lemma.

We will now show that the set $\Omega_{\tilde{\delta}}$ of fixed points of the morphism $\tilde{\delta}: \mathbb{P}^{1} \rightarrow \mathbb{P}^{1}$ defined by $\tilde{\delta}([x: y])=\left[g_{1}(x, y): g_{2}(x, y)\right]$ is equal to $\Lambda$, which will conclude the proof. Note that it is contained in $\Lambda$ and invariant under the action of $H$, since $\tilde{\delta}$ is $H$-equivariant.

Let us write $g_{1}=\beta \tilde{g_{1}}$ and $g_{2}=\beta \tilde{g_{2}}$, where $\beta, \tilde{g_{1}}, \tilde{g_{2}}$ are homogeneous and $\tilde{g_{1}}, \tilde{g_{2}}$ have no common root in $\mathbb{P}^{1}$. Note that the set $\Omega_{\tilde{\delta}}$ is equal to the zero set of the homogeneous polynomial $\tilde{g_{1}} y-\tilde{g_{2}} x$. 
We claim that none of the $P_{i}$ divides $\beta$. Indeed, otherwise such a $P_{i}$ would divide both $g_{1}$ and $g_{2}$ and thus also $f_{i, 1} \prod_{j \neq i} P_{j}$ and $f_{i, 2} \prod_{j \neq i} P_{j}$. Since $P_{i}$ has no common root with any of the $P_{j}$, this would imply that $P_{i}$ divides $f_{i, 1}$ and $f_{i, 2}$. This is impossible, since $P_{i}=f_{i, 1} y-f_{i, 2} x$, hence $P_{i}$ has degree bigger than $f_{i, 1}$ and $f_{i, 2}$.

Therefore, it follows from the equalities

$$
\prod_{i=1}^{r} P_{i}=g_{1} y-g_{2} x=\beta\left(\tilde{g_{1}} y-\tilde{g_{2}} x\right)
$$

that, for every index $i$, at least one point of $\Lambda_{i}$ is contained in $\Omega_{\tilde{\delta}}$. This latter set being invariant by $H$ and $\Lambda_{i}$ being an orbit under the action of $H$, we get that the whole set $\Lambda_{i}$ is contained in $\Omega_{\tilde{\delta}}$, for each $i=1 \ldots r$. This achieves the proof.

Corollary 4.4. Let $H \subset \operatorname{PGL}(2, \mathbb{C})=\operatorname{Aut}\left(\mathbb{P}^{1}\right)$ be a finite subgroup and let $\Lambda \subset \mathbb{P}^{1}$ be a finite subset. The following conditions are equivalent:

(1) The set $\Lambda$ is non-empty and invariant by $H$.

(2) There exists a $H$-equivariant morphism $\delta: \mathbb{P}^{1} \rightarrow \mathbb{P}^{1}$ such that

$$
\Lambda=\left\{q \in \mathbb{P}^{1} \mid \delta(q)=q\right\} .
$$

Proof. The implication (1) $\Rightarrow(2)$ follows directly from Proposition 4.3. Let us prove the other one.

Let $\delta: \mathbb{P}^{1} \rightarrow \mathbb{P}^{1}$ be a $H$-equivariant morphism whose fixed-point set is equal to $\Lambda$. The set $\Lambda$ is then invariant under the action of $H$, since $\delta(h(q))=h(\delta(q))=h(q)$ hold for all $h \in H$ and all $q \in \Lambda$.

Furthermore, let $f_{1}, f_{2} \in \mathbb{C}[x, y]$ be two homogeneous polynomials of the same degree and without common root in $\mathbb{P}^{1}$ such that $\delta([x: y])=\left[f_{1}(x, y): f_{2}(x, y)\right]$ for all points $[x: y] \in \mathbb{P}^{1}$. Since $\Lambda$ is the zero set of $f_{1} y-f_{2} x$, it is clearly non-empty.

\section{Equivariant embeddings into the affine three-space}

Let us recall that the following morphism

$$
\begin{aligned}
\mathbb{P}^{1} \times \mathbb{P}^{1} & \hookrightarrow \mathbb{P}^{3} \\
\left(\left[y_{0}: y_{1}\right],\left[z_{0}: z_{1}\right]\right) & \mapsto\left[y_{0} z_{0}: y_{0} z_{1}: y_{1} z_{0}: y_{1} z_{1}\right]
\end{aligned}
$$

is a classical closed embedding of $\mathbb{P}^{1} \times \mathbb{P}^{1}$ into $\mathbb{P}^{3}$ and that it induces an isomorphism between $\mathbb{P}^{1} \times \mathbb{P}^{1}$ and the quadric in $\mathbb{P}^{3}$ defined by the equation $x_{0} x_{3}=x_{1} x_{2}$. Moreover, since this embedding is canonical (it is given by the linear system $\left|-\frac{1}{2} K_{\mathbb{P}^{1} \times \mathbb{P}^{1}}\right|$ ), every automorphism of $\mathbb{P}^{1} \times \mathbb{P}^{1}$ extends to a unique automorphism of $\mathbb{P}^{3}$.

Identifying $\mathbb{A}^{3}$ with the complement in $\mathbb{P}^{3}$ of the hyperplane defined by the equation $x_{1}=x_{2}$, we obtain a closed embedding $\left(\mathbb{P}^{1} \times \mathbb{P}^{1}\right) \backslash \Delta \hookrightarrow \mathbb{A}^{3}$, where $\Delta$ denotes the diagonal curve $\Delta=\left\{(q, q) \mid q \in \mathbb{P}^{1}\right\} \subset \mathbb{P}^{1} \times \mathbb{P}^{1}$.

Consider the diagonal action of $\operatorname{PGL}(2, \mathbb{C})=\operatorname{Aut}\left(\mathbb{P}^{1}\right)$ on $\mathbb{P}^{1} \times \mathbb{P}^{1}$. Note that each automorphism of $\mathbb{P}^{1} \times \mathbb{P}^{1}$ coming from this action extends to an automorphism of $\mathbb{P}^{3}$ which preserves the plane of equation $x_{1}=x_{2}$. This yields an action of $\operatorname{PGL}(2, \mathbb{C})$ on $\mathbb{A}^{3}$ for which the closed embedding $\left(\mathbb{P}^{1} \times \mathbb{P}^{1}\right) \backslash \Delta \hookrightarrow \mathbb{A}^{3}$, that we defined above, becomes $\mathrm{PGL}(2, \mathbb{C})$-equivariant.

After a change of coordinates in $\mathbb{A}^{3}$, we obtain a $\operatorname{PGL}(2, \mathbb{C})$-equivariant embedding of $\left(\mathbb{P}^{1} \times \mathbb{P}^{1}\right) \backslash \Delta$ into $\mathbb{A}^{3}$, where the action of $\operatorname{PGL}(2, \mathbb{C})$ on $\mathbb{A}^{3}$ is linear. 
Lemma 5.1. The morphism

$$
\begin{aligned}
\iota: \quad\left(\mathbb{P}^{1} \times \mathbb{P}^{1}\right) \backslash \Delta & \hookrightarrow \mathbb{A}^{3} \\
\left(\left[y_{0}: y_{1}\right],\left[z_{0}: z_{1}\right]\right) & \mapsto\left(\frac{y_{0} z_{1}+y_{1} z_{0}}{y_{0} z_{1}-y_{1} z_{0}}, \frac{2 y_{0} z_{0}}{y_{0} z_{1}-y_{1} z_{0}}, \frac{2 y_{1} z_{1}}{y_{0} z_{1}-y_{1} z_{0}}\right)
\end{aligned}
$$

is a closed embedding whose image is the hypersurface of $\mathbb{A}^{3}$ defined by the equation $y z=x^{2}-1$.

Moreover, $\iota$ is $\operatorname{PGL}(2, \mathbb{C})$-equivariant, when we consider the actions of $\operatorname{PGL}(2, \mathbb{C})$ on $\left(\mathbb{P}^{1} \times \mathbb{P}^{1}\right) \backslash \Delta$ and $\mathbb{A}^{3}$ defined by

$$
\begin{array}{rll}
\mathrm{PGL}(2, \mathbb{C}) \times\left(\mathbb{P}^{1} \times \mathbb{P}^{1}\right) \backslash \Delta & \rightarrow & \left(\mathbb{P}^{1} \times \mathbb{P}^{1}\right) \backslash \Delta \\
\left(\left(\begin{array}{ll}
a & b \\
c & d
\end{array}\right),\left(\left[y_{0}: y_{1}\right],\left[z_{0}: z_{1}\right]\right)\right) & \mapsto & \left(\left[a y_{0}+b y_{1}: c y_{0}+d y_{1}\right],\left[a z_{0}+b z_{1}: c z_{0}+d z_{1}\right]\right)
\end{array}
$$

and

$$
\begin{gathered}
\operatorname{PGL}(2, \mathbb{C}) \times \mathbb{A}^{3} \rightarrow \mathbb{A}^{3} \\
\left(\left(\begin{array}{ll}
a & b \\
c & d
\end{array}\right),\left(\begin{array}{l}
x \\
y \\
z
\end{array}\right)\right) \mapsto \frac{1}{a d-b c}\left(\begin{array}{ccc}
a d+b c & a c & b d \\
2 a b & a^{2} & b^{2} \\
2 c d & c^{2} & d^{2}
\end{array}\right) \cdot\left(\begin{array}{l}
x \\
y \\
z
\end{array}\right) .
\end{gathered}
$$

Proof. Let $Q$ denotes the quadric hypersurface of $\mathbb{A}^{3}$ defined by the equation $y z=$ $x^{2}-1$. One checks that the morphism $\iota$ induces an isomorphism between $\left(\mathbb{P}^{1} \times \mathbb{P}^{1}\right) \backslash \Delta$ and $Q$ whose inverse morphism is given by

$$
\begin{aligned}
Q & \rightarrow \\
(x, y, z) & \mapsto \begin{cases}\left(\mathbb{P}^{1} \times \mathbb{P}^{1}\right) \backslash \Delta \\
([x+1: z],[y: x+1]) & \text { if } x \neq-1, \\
([y: x-1],[x-1: z]) & \text { if } x \neq 1 .\end{cases}
\end{aligned}
$$

It is also straightforward to check that $\iota$ is $\operatorname{PGL}(2, \mathbb{C})$-equivariant for the given actions.

Combining the latter lemma with the results of the previous section, we finally get Aut $(\Gamma)$-equivariant embeddings of every smooth affine rational curve $\Gamma$ into $\mathbb{A}^{3}$.

Theorem 5.2. For every rational smooth affine curve $\Gamma$, there exist a linear action of $\operatorname{Aut}(\Gamma)$ on $\mathbb{A}^{3}$ and a closed embedding $\tau: \Gamma \hookrightarrow \mathbb{A}^{3}$ which is $\operatorname{Aut}(\Gamma)$-equivariant for this action.

Proof. If $\Gamma=\mathbb{A}^{1}$, it suffices to consider the embedding $\tau: \mathbb{A}^{1} \rightarrow \mathbb{A}^{3}$ defined by $\tau(t)=(t, 0,0)$, and to let $\operatorname{Aut}(\Gamma)=\left\{x \mapsto a x+b \mid a \in \mathbb{C}^{*}, b \in \mathbb{C}\right\}$ act on $\mathbb{A}^{3}$ via the maps $(x, y, z) \mapsto(a x+b(y+1), y, z)$.

If $\Gamma=\mathbb{C}^{*}$, we consider the embedding $\tau: \Gamma \rightarrow \mathbb{A}^{3}$ defined by $\tau(t)=(t, 1 / t, 0)$. Its image is the curve in $\mathbb{A}^{3}$ defined by the equations $z=0$ and $x y=1$. Recall that

$$
\operatorname{Aut}(\Gamma)=\left\{\varphi_{\lambda}: x \mapsto \lambda x \mid \lambda \in \mathbb{C}^{*}\right\} \cup\left\{\psi_{\lambda}: x \mapsto \lambda x^{-1} \mid \lambda \in \mathbb{C}^{*}\right\} .
$$

The embedding $\tau$ becomes Aut $(\Gamma)$-equivariant, when we let Aut $(\Gamma)$ act on $\mathbb{A}^{3}$ via the maps $\Phi_{\lambda}:(x, y, z) \mapsto\left(\lambda x, \lambda^{-1} y, z\right)$ and $\Psi_{\lambda}:(x, y, z) \mapsto\left(\lambda y, \lambda^{-1} x, z\right)$.

If $\Gamma$ is equal to $\mathbb{P}^{1} \backslash \Lambda$, where $\Lambda$ is a finite set of at least 3 points, then its automorphism group $H=\operatorname{Aut}(\Gamma)$ is the finite subgroup of $\operatorname{PGL}(2, \mathbb{C})=\operatorname{Aut}\left(\mathbb{P}^{1}\right)$ that preserves the set $\Lambda$. Applying Corollary 4.4 let $\delta: \mathbb{P}^{1} \rightarrow \mathbb{P}^{1}$ be a $H$-equivariant morphism such that $\Lambda=\left\{q \in \mathbb{P}^{1} \mid \delta(q)=q\right\}$. This allows us to define a closed embedding $\hat{\tau}: \Gamma \rightarrow\left(\mathbb{P}^{1} \times \mathbb{P}^{1}\right) \backslash \Delta$ by letting $\hat{\tau}(q)=(q, \delta(q))$ for all $q \in \Gamma=\mathbb{P}^{1} \backslash \Lambda$. The morphism $\hat{\tau}$ is moreover $H$-equivariant, when $H$ acts diagonally on $\left(\mathbb{P}^{1} \times \mathbb{P}^{1}\right) \backslash \Delta$. 
Composing $\hat{\tau}$ with the $\mathrm{PGL}(2, \mathbb{C})$-equivariant closed embedding $\iota:\left(\mathbb{P}^{1} \times \mathbb{P}^{1}\right) \backslash \Delta \hookrightarrow$ $\mathbb{A}^{3}$ that we defined in Lemma [5.1] we obtain a closed embedding $\tau: \Gamma \rightarrow \mathbb{A}^{3}$ which is $H$-equivariant, as desired.

\section{Explicit formulas for the equivariant embeddings into $\mathbb{A}^{3}$}

The proof of Theorem 5.2 is constructive and already contains explicit Aut $(\Gamma)$ equivariant embeddings into $\mathbb{A}^{3}$ for the curves $\Gamma=\mathbb{A}^{1}$ and $\Gamma=\mathbb{A}^{1} \backslash\{0\}$. Let us now describe the construction for the other cases, i.e., when the automorphism group $\operatorname{Aut}(\Gamma)$ is finite.

We consider the curves $\Gamma=\mathbb{P}^{1} \backslash \Lambda$, where $\Lambda$ is a set of at least 3 points of $\mathbb{P}^{1}$. Let us denote by $H$ the subgroup of $\operatorname{Aut}\left(\mathbb{P}^{1}\right)=\operatorname{PGL}(2, \mathbb{C})$ that restricts to $\operatorname{Aut}(\Gamma)$, and denote as before by $G$ its pull-back in $\operatorname{SL}(2, \mathbb{C})$, which is a finite group of order $2|H|$. The set $\Lambda$ decomposes into $r$ orbits $\Lambda=\bigcup_{i=1}^{r} \Lambda_{i}$ of $H$. An orbit $\Lambda_{i}$ of $H$ is given by the zero set of a homogeneous polynomial $p_{i} \in \mathbb{C}[x, y]$. Some power $P_{i}=p_{i}^{d_{i}}$ of $p_{i}$ is invariant by the action of $G$ on $\mathbb{P}^{1}$ defined in Section 4 . For each $i$, Lemma 4.2 yields the existence of a $G$-invariant pair $\left(f_{i, 1}, f_{i, 2}\right) \in \operatorname{End}\left(\mathbb{A}^{2}\right)$ which satisfy $f_{i, 1} y-$ $f_{i, 2} x=P_{i}$. The $H$-equivariant morphism $\delta: \mathbb{P}^{1} \rightarrow \mathbb{P}^{1}$ given by Proposition 4.3 (or Corollary 4.4) is thus $\delta:[x: y] \rightarrow\left[f_{1}(x, y): f_{2}(x, y)\right]$, where

$$
f_{1}=\frac{1}{r}\left(\prod_{i=1}^{r} P_{i}\right) \sum_{i=1}^{r} \frac{f_{i, 1}}{P_{i}} \quad \text { and } \quad f_{2}=\frac{1}{r}\left(\prod_{i=1}^{r} P_{i}\right) \sum_{i=1}^{r} \frac{f_{i, 2}}{P_{i}} .
$$

Moreover, $\left(f_{1}, f_{2}\right)$ is invariant by $G$ and satisfies $f_{1} y-f_{2} x=\prod_{i=1}^{r} P_{i}$.

Following the proof of Theorem 5.2, we define a closed embedding $\Gamma=\mathbb{P}^{1} \backslash \Lambda \rightarrow$ $\left(\mathbb{P}^{1} \times \mathbb{P}^{1}\right) \backslash \Delta$ by $[x: y] \mapsto\left([x: y],\left[f_{1}: f_{2}\right]\right)$. We compose then this latter with the embedding $\iota:\left(\mathbb{P}^{1} \times \mathbb{P}^{1}\right) \backslash \Delta \rightarrow \mathbb{A}^{3}$ defined by Lemma [5.1, and obtain the following $\operatorname{Aut}(\Gamma)=H$-equivariant closed embedding of $\Gamma$ into $\mathbb{A}^{3}$.

$$
\begin{aligned}
\Gamma=\mathbb{P}^{1} \backslash \Lambda & \rightarrow \mathbb{A}^{3} \\
{[x: y] } & \mapsto\left(\frac{1}{r} \sum_{i=1}^{r} \frac{x f_{i, 2}+y f_{i, 1}}{x f_{i, 2}-y f_{i, 1}}, \frac{1}{r} \sum_{i=1}^{r} \frac{2 x f_{i, 1}}{x f_{i, 2}-y f_{i, 1}}, \frac{1}{r} \sum_{i=1}^{r} \frac{2 y f_{i, 2}}{x f_{i, 2}-y f_{i, 1}}\right) .
\end{aligned}
$$

So it suffices to determine the polynomials $f_{i, 1}$ and $f_{i, 2}$, which depend on $H$ and $\Lambda$, to get explicit embeddings.

Recall that any finite subgroup of $\operatorname{Aut}\left(\mathbb{P}^{1}\right)=\operatorname{PGL}(2, \mathbb{C})$ is isomorphic to $\mathbb{Z} / n \mathbb{Z}$ (the cyclic group of order $n$ ), $D_{2 n}$ (the dihedral group of order $2 n$ ), $\mathfrak{A}_{4}$ (the tetrahedral group), $\mathfrak{S}_{4}$ (the octahedral or cubic group) or $\mathfrak{A}_{5}$ (the icosahedral or dodecahedral group) and that there is only one conjugacy class for each of these groups (see e.g. 1]).

1) In the cyclic case, we can assume that $H \subset \operatorname{PGL}(2, \mathbb{C})$ is generated by $[x$ : $y] \mapsto\left[\xi_{n} x: y\right]$, where $\xi_{n}$ is a primitive $n$-th root of unity. Its pullback $G \subset \operatorname{SL}(2, \mathbb{C})$ is then generated by $\left(\begin{array}{cc}\zeta & 0 \\ 0 & \zeta^{-1}\end{array}\right)$, where $\zeta$ is a primitive $2 n$-th root of unity. An orbit $\Lambda_{i}$ of $H$ is given by the zero set of a polynomial $p_{i}=a_{i} x^{n}+b_{i} y^{n}$ for some $\left(a_{i}, b_{i}\right) \in \mathbb{C}^{2} \backslash\{(0,0)\}$ (the cases where $a_{i}=0$ or $b_{i}=0$ provide a fixed point with multiplicity $n)$. We thus get

$$
P_{i}=\left(p_{i}\right)^{2} \in \mathcal{O}\left(\mathbb{A}^{2}\right)^{G}
$$


and

$$
\left(f_{i, 1}, f_{i, 2}\right)=\left(b_{i} y^{n-1}\left(a_{i} x^{n}+b_{i} y^{n}\right),-a_{i} x^{n-1}\left(a_{i} x^{n}+b_{i} y^{n}\right)\right) \in \operatorname{End}\left(\mathbb{A}^{2}\right)^{G}
$$

which satisfy $f_{i, 1} y-f_{i, 2} x=P_{i}$ (note that the $f_{i, 1}$ and $f_{i, 2}$ are here not unique, and could also be chosen without common factor). The corresponding embedding $\Gamma=\mathbb{P}^{1} \backslash \Lambda \rightarrow \mathbb{A}^{3}$ is given by

$$
[x: y] \mapsto\left(\begin{array}{c}
\frac{1}{r} \sum_{i=1}^{r} \frac{a_{i} x^{n}-b_{i} y^{n}}{a_{i} x^{n}+b_{i} y^{n}} \\
\frac{1}{r} \sum_{i=1}^{r} \frac{-2 b_{i} x y^{n-1}}{a_{i} x^{n}+b_{i} y^{n}} \\
\frac{1}{r} \sum_{i=1}^{r} \frac{2 a_{i} x^{n-1} y}{a_{i} x^{n}+b_{i} y^{n}}
\end{array}\right) .
$$

2) In the dihedral case, we can assume that $H$ is generated by the maps $[x: y] \mapsto$ $\left[\xi_{n} x: y\right]$ and $[x: y] \mapsto[y: x]$. So $G$ is generated by $\left(\begin{array}{cc}\zeta & 0 \\ 0 & \zeta^{-1}\end{array}\right)$ and $\left(\begin{array}{cc}0 & \mathbf{i} \\ \mathbf{i} & 0\end{array}\right)$, where $\mathbf{i}$ denotes the imaginary unit $\sqrt{-1}$.

An orbit $\Lambda_{i}$ of $H$ is given by the zero set of $p_{i}=a_{i}\left(x^{2 n}+y^{2 n}\right)+2 b_{i} x^{n} y^{n}$ for some $\left(a_{i}, b_{i}\right) \in \mathbb{C}^{2} \backslash\{(0,0)\}$ and we thus get

$$
P_{i}=\left(p_{i}\right)^{2} \in \mathcal{O}\left(\mathbb{A}^{2}\right)^{G}
$$

and

$$
\left(f_{i, 1}, f_{i, 2}\right)=\left(y^{n-1}\left(b_{i} x^{n}+a_{i} y^{n}\right) p_{i},-x^{n-1}\left(a_{i} x^{n}+b_{i} y^{n}\right) p_{i}\right) \in \operatorname{End}\left(\mathbb{A}^{2}\right)^{G}
$$

which satisfy $f_{i, 1} y-f_{i, 2} x=P_{i}$ (note that $P_{i}=p_{i}$ is also possible if $n$ is even, and that as before the polynomials $f_{i, 1}, f_{i, 2}$ are not unique, and could also be chosen without common factor). This leads to the embedding $\Gamma=\mathbb{P}^{1} \backslash \Lambda \rightarrow \mathbb{A}^{3}$ defined by

$$
[x: y] \mapsto\left(\begin{array}{c}
\frac{1}{r} \sum_{i=1}^{r} \frac{a_{i}\left(x^{2 n}-y^{2 n}\right)}{a_{i}\left(x^{2 n}+y^{2 n}\right)+2 b_{i} x^{n} y^{n}} \\
\frac{1}{r} \sum_{i=1}^{r} \frac{-2 x y^{n-1}\left(b_{i} x^{n}+a_{i} y^{n}\right)}{a_{i}\left(x^{2 n}+y^{2 n}\right)+2 b_{i} x^{n} y^{n}} \\
\frac{1}{r} \sum_{i=1}^{r} \frac{2 x^{n-1} y\left(a_{i} x^{n}+b_{i} y^{n}\right)}{a_{i}\left(x^{2 n}+y^{2 n}\right)+2 b_{i} x^{n} y^{n}}
\end{array}\right) .
$$

3) In the case of the tetrahedral group, we can assume that $H \cong \mathfrak{A}_{4}$ is generated by the maps $[x: y] \mapsto[\mathbf{i}(x+y): x-y]$ and $[x: y] \mapsto[x:-y]$. This implies that $G$ is generated by $\frac{1}{2}\left(\begin{array}{rr}\mathbf{i}-1 & \mathbf{i}-1 \\ \mathbf{i}+1 & -\mathbf{i}-1\end{array}\right)$ and $\left(\begin{array}{rr}-\mathbf{i} & 0 \\ 0 & \mathbf{i}\end{array}\right)$. An orbit $\Lambda_{i}$ of $H$ is given by the zero set of

$$
p_{i}=6 a_{i}\left(x^{5} y-x y^{5}\right)^{2}+b_{i}\left(x^{4}+y^{4}\right)\left(x^{8}+y^{8}-34 x^{4} y^{4}\right),
$$


for some $\left(a_{i}, b_{i}\right) \in \mathbb{C}^{2} \backslash\{(0,0)\}$. We thus get

$$
\begin{aligned}
P_{i} & =p_{i} \in \mathcal{O}\left(\mathbb{A}^{2}\right)^{G} \\
f_{i, 1} & =a_{i}\left(x^{10} y-6 x^{6} y^{5}+5 x^{2} y^{9}\right)+b_{i}\left(-11 x^{8} y^{3}-22 x^{4} y^{7}+y^{11}\right) \\
f_{i, 2} & =-a_{i}\left(5 x^{9} y^{2}-6 x^{5} y^{6}+x y^{10}\right)-b_{i}\left(x^{11}-22 x^{7} y^{4}-11 x^{3} y^{8}\right)
\end{aligned}
$$

which satisfy $\left(f_{i, 1}, f_{i, 2}\right) \in \operatorname{End}\left(\mathbb{A}^{2}\right)^{G}$ and $f_{i, 1} y-f_{i, 2} x=P_{i}$ as before. This gives the embedding $\Gamma=\mathbb{P}^{1} \backslash \Lambda \rightarrow \mathbb{A}^{3}$ defined by

$$
[x: y] \mapsto\left(\begin{array}{l}
\frac{1}{r} \sum_{i=1}^{r} \frac{4 a_{i} x^{2} y^{2}\left(x^{4}+y^{4}\right)\left(x^{4}-y^{4}\right)+b_{i}\left(x^{12}-11 x^{8} y^{4}+11 x^{4} y^{8}-y^{12}\right)}{6 a_{i}\left(x^{5} y-x y^{5}\right)^{2}+b_{i}\left(x^{4}+y^{4}\right)\left(x^{8}+y^{8}-34 x^{4} y^{4}\right)} \\
\frac{1}{r} \sum_{i=1}^{r} \frac{-2 x\left(a_{i}\left(x^{10} y-6 x^{6} y^{5}+5 x^{2} y^{9}\right)+b_{i}\left(-11 x^{8} y^{3}-22 x^{4} y^{7}+y^{11}\right)\right)}{6 a_{i}\left(x^{5} y-x y^{5}\right)^{2}+b_{i}\left(x^{4}+y^{4}\right)\left(x^{8}+y^{8}-34 x^{4} y^{4}\right)} \\
\frac{1}{r} \sum_{i=1}^{r} \frac{2 y\left(a_{i}\left(5 x^{9} y^{2}-6 x^{5} y^{6}+x y^{10}\right)+b_{i}\left(x^{11}-22 x^{7} y^{4}-11 x^{3} y^{8}\right)\right)}{6 a_{i}\left(x^{5} y-x y^{5}\right)^{2}+b_{i}\left(x^{4}+y^{4}\right)\left(x^{8}+y^{8}-34 x^{4} y^{4}\right)}
\end{array}\right) .
$$

It is also possible to describe similarly the other cases $\left(\mathfrak{S}_{4}\right.$ and $\left.\mathfrak{A}_{5}\right)$, but the formulas are even more intricate.

\section{References}

[1] A. Beauville, Finite subgroups of $\mathrm{PGL}_{2}(K)$, in Vector bundles and complex geometry, Vol. 522 of Contemp. Math., 23-29, Amer. Math. Soc., Providence, RI (2010).

[2] A. Borel, Linear algebraic groups, Vol. 126 of Graduate Texts in Mathematics, Springer-Verlag, New York, second edition (1991), ISBN 0-387-97370-2.

[3] G. E. Bredon, Introduction to compact transformation groups, Academic Press, New YorkLondon (1972). Pure and Applied Mathematics, Vol. 46.

[4] H. Derksen, F. Kutzschebauch, and J. Winkelmann, Subvarieties of $\mathbf{C}^{n}$ with non-extendable automorphisms, J. Reine Angew. Math. 508 (1999) 213-235.

[5] S. Friedland and J. Milnor, Dynamical properties of plane polynomial automorphisms, Ergodic Theory Dynam. Systems 9 (1989), no. 1, 67-99.

[6] M. Furushima, Finite groups of polynomial automorphisms in $\mathbf{C}^{n}$, Tohoku Math. J. (2) 35 (1983), no. 3, 415-424.

[7] L. Greenberg, Maximal Fuchsian groups, Bull. Amer. Math. Soc. 69 (1963) 569-573.

[8] S. Kaliman, Extensions of isomorphisms between affine algebraic subvarieties of $k^{n}$ to automorphisms of $k^{n}$, Proc. Amer. Math. Soc. 113 (1991), no. 2, 325-334.

[9] T. Petrie and J. D. Randall, Finite-order algebraic automorphisms of affine varieties, Comment. Math. Helv. 61 (1986), no. 2, 203-221.

[10] A. Sathaye, On planar curves, Amer. J. Math. 99 (1977), no. 5, 1105-1135.

[11] J.-P. Serre, Trees, Springer-Verlag, Berlin-New York (1980), ISBN 3-540-10103-9. Translated from the French by John Stillwell.

[12] V. Srinivas, On the embedding dimension of an affine variety, Math. Ann. 289 (1991), no. 1, $125-132$. 
J. Blanc, Universität Basel, Mathematisches Institut, Rheinsprung 21, CH-4051 Basel, SWITZERLAND.

E-mail address: jeremy.blanc@unibas.ch

$U R L:$ http://jones.math.unibas.ch/ blanc/

J.-P. Furter, Dpt. of Math., Univ. of La Rochelle, av. Crépeau, 17000 La Rochelle, FRANCE

E-mail address: jpfurter@univ-lr.fr

$U R L:$ http://perso.univ-lr.fr/jpfurter/

P.-M. Poloni, Universität Basel, Mathematisches Institut, Rheinsprung 21, CH-4051 Basel, Switzerland

E-mail address: pierre-marie.poloni@unibas.ch

URL: http://jones.math.unibas.ch/ poloni/ 